\title{
Integração da Psicologia e Odontologia na DTM: revisão sistematizada
}

\author{
Integration of Psychology and Dentistry in TMD: a systematized review \\ Integración de la Psicología y Odontología en la DTM: \\ una revisión sistematizada \\ Adriana Cristina ZAVANELLI ${ }^{1}$ \\ Maria Cristina Rosifini ALVES REZENDE ${ }^{1}$ \\ Otavio Marino dos SANTOS-NETO ${ }^{2}$ \\ Renato Salviato FAJARDO ${ }^{1}$ \\ ${ }^{l}$ Departamento de Materiais Odontológicos e Prótese, Faculdade de Odontologia, UNESP Univ. Estadual Paulista, \\ 16015-050 Araçatuba - SP, Brasil \\ ${ }^{2}$ Mestrando em Odontologia (Reabilitação Oral) pela Faculdade Odontologia de Ribeirão Preto, USP, Univ. de São Paulo,
} 14090-904 Ribeirão Preto-SP, Brasil

\begin{abstract}
Resumo
A etiologia mais provável da disfunção temporomandibular (DTM) é multifatorial. Esse fato torna complexo o seu diagnóstico e tratamento para a área de Odontologia. Um dos componentes importantes na etiologia e perpetuação da DTM, ainda pouco explorado no tratamento dessa síndrome, é o psicológico. Sendo assim, este artigo teve como objetivo apresentar revisão sistematizada da literatura enfocando a integração da DTM e psicologia. Foi realizado um levantamento nas bases de dados Pubmed e Scielo no período de 2010 a 2017 utilizando como critério de inclusão: artigos publicados na língua inglesa ou portuguesa com os descritores psicologia e síndrome da articulação temporomandibular ou psicoterapia e articulação temporomandibular. Foram excluídos os artigos onde o componente psicológico nos casos de DTM foi apenas citado e não avaliado e artigos de revisão de literatura. A busca selecionou 23 artigos, dos quais 17 citavam a psicologia como etiologia, relacionado com a qualidade de vida e somente 4 propuseram terapias psicológicas para o tratamento da DTM, sendo que 1 associou a psicoterapia com o relaxamento. Os resultados obtidos enfatizaram a importância da integração entre as áreas do saber para proporcionar atendimento integral aos portadores desta disfunção. Apesar disso, a literatura disponível abordando terapias psicológicas no tratamento da DTM é escassa justificando a necessidade de estudos nessa área.

Descritores: Síndrome da Disfunção da Articulação Temporomandibular, Odontologia, Psicoterapia.
\end{abstract}

\section{Abstract}

The more probably etiology of the temporomandibular dysfunction is multifactorial. This fact makes complex their diagnosis and treatment for Dentistry. One the importants components in the etiology and perpetuation of the TMD, still little explored in the treatment this syndrome is the psychological component. Therefore, this article aimed at the systematized review of the literature focusing on the integration of TMD and psychology. A survey was carried in the databases Pubmed and Scielo in the period between 2010 and 2017 using like include criteria: articles published in English or Portuguese language with the descriptors psychology and temporomandibular join syndrome or psychoteraphy and temporomandibular join. A survey was carried out in Pubmed and Scielo databases from 2010 to 2017 using as inclusion criterion: articles published in English or Portuguese with the keywords psychology / temporomandibular joint syndrome, psychotherapy / temporomandibular joint. We excluded the articles where the psychological component in cases of TMD was only cited and not evaluated and review of literature articles. The search selected 23 articles, of which 17 cited psychology as etiology, related to quality of life and only 4 proposed psychological therapies for the treatment of TMD, 1 of which associated psychotherapy with relaxation. The results obtained emphasized the importance of integration among the knowledge areas to provide integral care to the patients with this dysfunction. Despite this, the available literature addressing psychological therapies in the treatment of TMD is scarce, justifying the need for studies in this area. Descriptors: Temporomandibular Joint Dysfunction Syndrome; Dentistry; Psychotherapy.

\section{Resumen}

La más probable etiología de la disfunción temporomandibular (DTM) es multifactorial. Ese facto torna complejo su diagnóstico y tratamiento para el área de Odontología. Uno de los componentes importantes en la etiología y perpetuación de la DTM, aún poco explorado en el tratamiento de esa síndrome, es lo psicológico. Por lo tanto, este artigo tuvo como objetivo presentar una revisión sistematizada de la literatura enfocando la integración de la DTM y la psicología. Fue realizado un levantamiento en las bases de dados Pubmed y Scielo en lo período de 2010 a 2017 utilizando como criterio de inclusión: artículos publicados en la lengua inglesa o portuguesa con las palabras-claves psicología /síndrome de la articulación temporomandibular, psicoterapia/ articulación temporomandibular. Fueron excluidos los artículos donde el componente psicológico en los casos de DTM fue apenas citado y no evaluado y artículos de revisión de literatura. La busca seleccionó 23 artículos, de los cuales 17 citaban la psicología como etiología, relacionado con la cualidad de vida y solamente 4 propusieran terapias psicológicas para el tratamiento de la DTM, siendo que 1 asoció la psicoterapia con el relajamiento. Los resultados obtenidos enfatizaron la importancia de la integración entre las áreas do saber para proporcionar atendimiento integral a los portadores de esta disfunción. Sin embargo, la literatura disponible abordando las terapias psicológicas en el tratamiento de la DTM es escasa justificando la necesidad de estudios en esa área.

Descriptores: Síndrome de la Disfunción de Articulación Temporomandibular; Odontología; Psicoterapia.

\section{INTRODUÇÃO}

A Disfunção da Articulação Temporomandibular (DTM) é identificada de acordo com os Critérios de Diagnóstico e Pesquisa para DTM (CDP-DTM) podendo ser diagnosticada na presença de sinais e sintomas tais como dor e ruídos durante o movimento mandibular, limitação dos movimentos mandibulares, dificuldade em abrir a boca e parafunções oclusais.

A etiologia da DTM é diversa ${ }^{1}$; além de determinantes genéticos e ambientais, os fatores psicológicos também podem ser considerados como responsáveis. A ansiedade e depressão são estados psicológicos que podem induzir ou exacerbar a DTM ${ }^{2}$. Pode-se perceber que pessoas com dores crônicas sofrem estresse social mais exacerbado, quando comparadas às pessoas livres de dores crônicas ${ }^{3}$. As queixas das dores podem ser associadas a problemas musculoesqueléticos e até mesmo a somatização de ansiedade e depressão ${ }^{4}$.

Estudos recentes comprovaram que o gênero feminino prevalece em relação à busca de serviço especializado em dor orofacial e os pacientes atendidos apresentaram DTM e tensão emocional elevada ${ }^{5-6}$. Considerando-se a íntima correlação das alterações 
emocionais com a DTM, vários estudos sugerem que ao mesmo tempo em que a DTM provém de fatores emocionais, a própria DTM promove um aumento em transtornos emocionais. Desta forma, a relação com o sexo feminino está correlacionada com as alterações da autoestima, onde averiguar rotinas de tratamento humanizado pode favorecer o desenvolvimento do próprio tratamento da disfunção ${ }^{7}$.

Assim, os critérios de diagnóstico devem ser realizados com base no modelo biopsicossocial, onde embora as doenças de estresse mentais se mostrem exacerbadoras da disfunção, elas não são os únicos fatores da DTM. Portanto, exames diagnósticos psicológicos detalhados, bem como a avaliação psicossocial e psiquiátrica são recomendados em pacientes com DTM ${ }^{2,8}$.

Dentre os inúmeros fatores etiológicos da DTM observa-se importância da integração das áreas da saúde para que se possa proporcionar um tratamento integral, nas quais as alterações psicológicas como a ansiedade, o estresse, a depressão, hábitos orais autodestrutivos e a dor crônica apresentam-se em grandes escalas nos pacientes, sendo necessária a participação do psicólogo no diagnóstico, no tratamento e no desenvolvimento de pesquisa sobre o tema ${ }^{9,10}$.

Diante do conteúdo reunido na literatura, pode-se perceber a importância do componente psicológico na DTM. Desta maneira, este estudo realizou uma revisão sistematizada a fim de verificar possíveis correlações entre terapias cognitivos comportamentais (TCC) e o tratamento da DTM.

\section{MATERIAL E MÉTODO}

Trata-se de um estudo qualitativo realizado por meio de revisão sistematizada da literatura. Foram consultadas as bases de dados Scielo e PubMed sobre a intersecção da Psicologia e Odontologia, à procura de uma possível terapia proposta frente à DTM usando os termos psicologia, síndrome da articulação temporomandibular, psicoterapia, articulação temporomandibular. Os operadores boleanos "AND" e "OR" foram utilizados para combinar as palavras chaves. Os artigos selecionados para este estudo tiveram como critério de inclusão: trabalhos publicados nas línguas inglesa e/ou portuguesa, artigos que apresentam como tema central a DTM correlacionada com a psicologia, em busca de uma possível terapia proposta em suas conclusões; e estudos entre os anos de 2010 e 2017. Foram excluídos os artigos com ênfases em outras áreas que não se encaixavam na intersecção entre psicologia e DTM.

\section{RESULTADOS}

A busca selecionou dentro dos critérios de inclusão e exclusão 23 artigos, dos quais 17 citavam a psicologia como etiologia, relacionado com a qualidade de vida e somente 4 propuseram terapias psicológicas para o tratamento da DTM, sendo que 1 associou a psicoterapia com o relaxamento (Quadros 1 e 2).

Quadro 1. Artigos selecionados e aceitos segundo critério de inclusão com autores, ferramenta de avaliação utilizada, terapia proposta e conclusão

\begin{tabular}{|c|c|c|c|c|}
\hline Autor & $\begin{array}{l}\text { População } \\
\text { Avaliada }\end{array}$ & Ferramentas de Avaliação & $\begin{array}{l}\text { Terapia } \\
\text { Proposta }\end{array}$ & Principais Conclusões \\
\hline $\begin{array}{l}\text { Motta et al., } \\
2015^{9}\end{array}$, & $\begin{array}{l}3538 \text { adolescentes } \\
\text { entre } 10 \text { e } 19 \text { anos }\end{array}$ & $\begin{array}{l}\text { Para o grau de severidade da DTM: } \\
\text { Índice de Fonseca Para avaliar a } \\
\text { ansiedade; Inventário de Ansiedade } \\
\text { Traço Estado. }\end{array}$ & $\begin{array}{l}\text { Nenhuma } \\
\text { proposta. }\end{array}$ & $\begin{array}{l}\text { Estudo demonstra a relação entre a DTM e os níveis de } \\
\text { ansiedade em adolescentes, da cidade de São Roque - } \\
\text { SP, indicando que quanto maior o nível de ansiedade } \\
\text { maior a chance de desenvolver DTM. }\end{array}$ \\
\hline Litt et al., $2010^{11}$ & $\begin{array}{l}85 \text { mulheres e } 16 \\
\text { homens com queixa de } \\
\text { dor na região da ATM }\end{array}$ & $\begin{array}{l}\text {-Inventário multidirecional de dor } \\
\text {-Escala de estudos epidemiológicos } \\
\text { de depressão } \\
\text {-Questionário de mudanças no estágio } \\
\text { da dor }\end{array}$ & $\begin{array}{l}\text { Terapia Cognitivo- } \\
\text { Comportamental } \\
\text { (TCC) }\end{array}$ & $\begin{array}{l}\text { Breves tratamentos podem reduzir a dor, interferência e } \\
\text { sintomas depressivos em pacientes com DTM e a } \\
\text { adição de habilidades de enfrentamento cognitivo- } \\
\text { comportamental poderá trazer benefícios. }\end{array}$ \\
\hline $\begin{array}{l}\text { Kuroiwa et al., } \\
2011^{12}\end{array}$ & 91 pacientes & $\begin{array}{l}\text { Questionário Genérico de Avaliação } \\
\text { de Qualidade de Vida - Medical } \\
\text { OutcomesStudy } 36 \text { - Item Short } \\
\text { Health Survey (SF-36). Mental (SM), } \\
\text { aspectos emocionais (AE), aspectos } \\
\text { sociais (AS) e vitalidade (V). }\end{array}$ & $\begin{array}{l}\text { Nenhuma } \\
\text { proposta }\end{array}$ & $\begin{array}{l}\text { A qualidade de vida pode ser influenciada em pacientes } \\
\text { que apresentam dor orofacial ou DTM, com isso, pode- } \\
\text { se concluir que a qualidade de vida dos pacientes sofre } \\
\text { um impacto negativo, de acordo com os prejuízos físicos } \\
\text { e mentais analisados. }\end{array}$ \\
\hline $\begin{array}{l}\text { Fillingim et al., } \\
2011^{13}\end{array}$ & $\begin{array}{l}1.633 \text { pacientes (grupo } \\
\text { controle). } \\
185 \text { pacientes com } \\
\text { DTM. }\end{array}$ & $\begin{array}{lcc}\text { Avaliação } & \text { Prospectiva da } & \text { Dor } \\
\text { Orofacial e Avaliação de Riscos } & \end{array}$ & $\begin{array}{l}\text { Nenhuma } \\
\text { proposta; }\end{array}$ & $\begin{array}{l}\text { Há uma grande diferença entre o fator psicossocial de } \\
\text { pacientes com e sem dor, podendo acarretar no } \\
\text { desenvolvimento de DTM. }\end{array}$ \\
\hline $\begin{array}{l}\text { Calderon et al., } \\
2011^{14}\end{array}$ & 47 mulheres & $\begin{array}{l}\text {-RDCTDM } \\
\text {-Inventarário e depressão de beck s } \\
\text {-Perfil do impacto de saúde oral } \\
\text { modificado para a dor orofacial } \\
\text {-Índice de qualidade de sono de } \\
\text { Pittsburg }\end{array}$ & $\begin{array}{ll}\text { Amitriptilina } & \text { e TCC; } \\
\text { placebo e } & \text { TCC; e } \\
\text { placebo } & \\
7 & \text { semanas } \\
\text { consecutivas } & \end{array}$ & $\begin{array}{l}\text { A combinação de amitriptilina e TCC pode ser eficaz na } \\
\text { redução dos níveis de dor e depressãobem como na } \\
\text { melhoria da qualidade de vida e de sono em pacientes. }\end{array}$ \\
\hline $\begin{array}{l}\text { Aggarwal al., } \\
2011^{15}\end{array}$ & $\begin{array}{l}\text { Adultos com dores } \\
\text { orofaciais crônicas. }\end{array}$ & Bases de dados eletrônicos & $\begin{array}{l}\text { Terapia } \\
\text { comportamental } \\
\text { cognitiva }\end{array}$ & $\begin{array}{l}\text { O estudo aponta a necessidade da intervenção } \\
\text { psicossocial para o tratamento de dores orofaciais } \\
\text { crônicas, como a DTM; em seu desfecho, o estudo } \\
\text { aponta a necessidade do desenvolvimento de pesquisas } \\
\text { unindo essas duas interfaces. }\end{array}$ \\
\hline $\operatorname{Kim}_{2012^{16}}$ et al., & $\begin{array}{l}36 \text { pacientes com DTM } \\
\text { com problemas } \\
\text { psicológicos }\end{array}$ & $\begin{array}{l}\text { Indice de gravidade dos sintomas } \\
\text { (SSI) e índice craniomandibular (CMI) }\end{array}$ & $\begin{array}{l}\text { Nenhuma } \\
\text { proposta. }\end{array}$ & Há necessidade de melhorias nesta área de estudo. \\
\hline $\begin{array}{l}\text { Miettinen et al., } \\
2012^{17}\end{array}$ & $\begin{array}{l}70 \text { pacientes (grupo } \\
\text { controle) } \\
79 \text { pacientes com DTM }\end{array}$ & $\begin{array}{l}\text { Critérios de diagnostico RDC/TMD. } \\
\text { Aplicação do questionário Oral Health } \\
\text { Impact Profile (OHIP-14). }\end{array}$ & $\begin{array}{l}\text { Nenhuma } \\
\text { proposta. }\end{array}$ & $\begin{array}{l}\text { O estudo conclui sobre a importância da interface } \\
\text { (estresse psicológico do indivíduo com a sua saúde } \\
\text { bucal e qualidade de vida), ligada com a somatização e } \\
\text { a depressão, reforçando o tratamento em conjunto e } \\
\text { precoce da DTM }\end{array}$ \\
\hline $\begin{array}{l}\text { Diniz et al., } \\
2012^{18}\end{array}$, & $\begin{array}{l}55 \text { formandos do } \\
\text { ensino médio. }\end{array}$ & $\begin{array}{l}\text { Questionário da Academia Americana } \\
\text { de Dor Orofacial (AAOP), Estresse de } \\
\text { Lipp para adultos Inventory (ISSL) e o } \\
\text { Inventário de Ansiedade Beck (BAI). }\end{array}$ & $\begin{array}{l}\text { Nenhuma } \\
\text { proposta. }\end{array}$ & $\begin{array}{l}\text { O estudo aponta a fragilidade psicológica de } \\
\text { vestibulandos, onde aumenta a pré-disposição para o } \\
\text { desenvolvimento de DTM, por conta do estresse. }\end{array}$ \\
\hline $\begin{array}{l}\text { Bezerra et al., } \\
2012^{19}\end{array}$ & 336 acadêmicos & $\begin{array}{l}\text { İndice Anamnésico } \\
\text { O Inventário de Ansiedade Traço- } \\
\text { Estado (IDATE). } \\
\text { Qui-quadrado de Pearson } \\
\text { Exato de Fisher (intervalo de } \\
\text { confiança de 95\%) }\end{array}$ & $\begin{array}{l}\text { Nenhuma } \\
\text { proposta }\end{array}$ & $\begin{array}{l}\text { Uma avaliação na incidência de DTM em acadêmicos, } \\
\text { com alta incidência em diversos cursos, porém com } \\
\text { maior incidência em alunos de Fisioterapia. }\end{array}$ \\
\hline $\begin{array}{l}\text { Zavanelli et al., } \\
2013^{20}\end{array}$ & $\begin{array}{l}9 \text { profissionais, } \\
\text { graduandos e pós- } \\
\text { graduandos. }\end{array}$ & $\begin{array}{l}\text { Entrevistas gravadas e transcritas na } \\
\text { integra. }\end{array}$ & $\begin{array}{l}\text { Nenhuma } \\
\text { proposta }\end{array}$ & $\begin{array}{l}\text { Levando-se em consideração a importância das } \\
\text { pesquisas da dor orofacial, o estudo teve como intuito } \\
\text { promover uma maior compreensão da visão dos } \\
\text { próprios cirurgiões-dentistas com relação à DTM. }\end{array}$ \\
\hline
\end{tabular}


Quadro 2. Artigos selecionados e aceitos segundo critério de inclusão com autores, ferramenta de avaliação utilizada, terapia proposta e conclusão

\begin{tabular}{|c|c|c|c|c|}
\hline Autor & $\begin{array}{l}\text { População } \\
\text { Avaliada }\end{array}$ & Ferramentas de Avaliação & $\begin{array}{l}\text { Terapia } \\
\text { Proposta }\end{array}$ & Principais Conclusões \\
\hline $\begin{array}{l}\text { Fillingim et al., } \\
2013^{21}\end{array}$ & 3263 pacientes & $\begin{array}{l}\text { Instrumentos psicológicos que avaliam } \\
\text { o ajuste geral psicológica e da } \\
\text { personalidade, angústia afetiva, } \\
\text { estresse psicossocial, sintomas } \\
\text { somáticos, e dor enfrentamento. } \\
\text { Criterios de diagnóstico RDC/TMD }\end{array}$ & $\begin{array}{l}\text { Nenhuma } \\
\text { proposta }\end{array}$ & $\begin{array}{l}\text { Este artigo relata que várias variáveis psicológicas } \\
\text { predizem a DTM. As medidas dos sintomas somáticos } \\
\text { foram mais fortemente associadas ao início das DTM, } \\
\text { mas o estresse percebido, os eventos da vida anterior e } \\
\text { o efeito negativo também prevêem a incidência de DTM. }\end{array}$ \\
\hline $\begin{array}{l}\text { Shedden et al., } \\
2013^{22}\end{array}$ & 58 pacientes com DTM & $\begin{array}{l}\text { Criterios de diagnóstico para DTM. } \\
\text { DSM-IV }\end{array}$ & $\begin{array}{l}\text { Terapia cognitivo } \\
\text { comportamental }\end{array}$ & $\begin{array}{l}\text { Avaliou-se a necessidade da terapia TCC para o auxílio } \\
\text { pessoal e no enfrentamento da dor, assinalando a } \\
\text { importância da psicologia dentro das clinicas de DTM }\end{array}$ \\
\hline $\begin{array}{l}\text { Kirschneck C et } \\
\text { al., } 2013^{23}\end{array}$ & $\begin{array}{l}100 \text { pacientes com dor } \\
\text { facial crônica e / ou dor } \\
\text { de cabeça com o } \\
\text { diagnóstico presuntivo } \\
\text { de (DTM) }\end{array}$ & $\begin{array}{l}\text {-Inventário de Dor (intensidade, } \\
\text { frequência, duração, interferência) } \\
\text {-Questionário de Dor McGill } \\
\text {-Lista de queixa de Zerssen } \\
\text {-Questionario de atitudes irracionais } \\
\text {-Questionário de comportamento da } \\
\text { dor }\end{array}$ & $\begin{array}{l}\text { Nenhuma } \\
\text { proposta. }\end{array}$ & $\begin{array}{l}\text { O relaxamento auto-administrado foi positivo sobre a } \\
\text { percepção da dor, no entanto, o benefício pode ser } \\
\text { maior em pacientes com maiores impacto dos sintomas. }\end{array}$ \\
\hline $\begin{array}{l}\text { Wieckiewicz et } \\
\text { al., } 2014^{24}\end{array}$ & 456 estudantes & Critérios de diagnósticos RDC/TMD & $\begin{array}{l}\text { Nenhuma } \\
\text { proposta }\end{array}$ & $\begin{array}{l}\text { O objetivo do estudo foi avaliar as correlações entre a } \\
\text { DTM e fatores psicoemocionais. O desfecho do estudo } \\
\text { concretizou que carga emocional e excitação excessiva } \\
\text { favorece o desenvolvimento de DTM, sendo assim } \\
\text { necessária a pesquisa nesta área. }\end{array}$ \\
\hline $\begin{array}{l}\text { Roldán-Barraza } \\
\text { et al., } 2014^{25}\end{array}$ & $\begin{array}{l}\text { Triagem e ensaios } \\
\text { clínicos aleatórios. }\end{array}$ & $\begin{array}{l}\text { Review programa de computador } \\
\text { Manager } \\
\text { Sistemática meta-analise }\end{array}$ & $\begin{array}{l}\text { Nenhuma } \\
\text { proposta }\end{array}$ & $\begin{array}{l}\text { Uma sistemática meta-análise para averiguar a } \\
\text { correlação entre a placa oclusal e fatores } \\
\text { psicoemocionais; verificando-se que nenhuma evidência } \\
\text { clinica foi encontrada para distinguir esses dois fatores } \\
\text { correlacionados. }\end{array}$ \\
\hline $\begin{array}{l}\text { Ismail et al., } \\
2015^{26}\end{array}$ & $\begin{array}{l}92 \text { doentes } \\
90 \text { controles }\end{array}$ & $\begin{array}{l}\text { Questionários sobre a dor subjetiva, } \\
\text { severidade da dor crônica, deficiência } \\
\text { maxilar, bem-estar emocional e } \\
\text { depressão, e um exame clínico. }\end{array}$ & $\begin{array}{l}\text { Nenhuma } \\
\text { proposta }\end{array}$ & $\begin{array}{l}\text { O estudo teve como objetivo acessar a prevalência de } \\
\text { depressão entre pacientes com DTM em comparação } \\
\text { com pacientes sem DTM. Desta maneira verificou-se } \\
\text { necessário um rastreio regular para problemas } \\
\text { psicológicos, usando questionários padronizados, } \\
\text { integrados no exame clínico de pacientes com DTM. }\end{array}$ \\
\hline $\begin{array}{l}\text { Costa } \\
2015^{27}\end{array}$ et al., & $\begin{array}{l}60 \text { adultos classificados } \\
\text { com dores miofasciais } \\
\text { mastigatória. }\end{array}$ & $\begin{array}{l}\text { Testes para análises estatísticas: } \\
\text { Two-way ANOVA, Friedman e Mann- } \\
\text { Whitney }\end{array}$ & $\begin{array}{l}\text { Nenhuma } \\
\text { proposta }\end{array}$ & $\begin{array}{l}\text { O estudo teve como objetivo averiguar a eficácia da } \\
\text { placa oclusal em pacientes com DTM. No final do } \\
\text { estudo pode-se identificar que a placa oclusal pode } \\
\text { facilitar a melhoria psicológica do paciente. }\end{array}$ \\
\hline $\begin{array}{l}\text { Salameh et al., } \\
2015^{28}\end{array}$ & $\begin{array}{l}120 \text { participantes }(60 \\
\text { com DTM e } 60 \\
\text { controle). Sujeitos na } \\
\text { faixa etária de } 19 \text { a } 44 \\
\text { anos, com } \\
\text { hábitos de sono } \\
\text { regulares, arcos } \\
\text { dentários completos. } \\
\text { O critério de inclusão } \\
\text { para pacientes com } \\
\text { DTM foi sintomas } \\
\text { clínicos presentes. }\end{array}$ & $\begin{array}{l}\text { Foram utilizados dois questionários } \\
\text { para avaliação do estresse: o PSS } 10 \\
\text { e a medida psicossocial dos Critérios } \\
\text { de Diagnóstico de Pesquisa (CDP). } \\
\text { Os níveis de cortisol salivar foram } \\
\text { medidos por um método colorimétrico } \\
\text { imunoenzimático competitivo. }\end{array}$ & $\begin{array}{l}\text { Nenhuma } \\
\text { proposta }\end{array}$ & $\begin{array}{l}\text { Este estudo concluiu que o estresse psicossocial } \\
\text { desempenha um papel importante na etiopatogenia da } \\
\text { DTM. As mulheres estão em maior risco de DTM } \\
\text { quando comparadas aos homens. Sub-tipos de } \\
\text { pacientes com DTM têm aproximadamente o mesmo } \\
\text { nível de estresse. Os distúrbios musculares foram os } \\
\text { mais comuns. }\end{array}$ \\
\hline $\begin{array}{l}\text { Tuuliainen et al., } \\
2015^{29}\end{array}$ & $\begin{array}{l}6155 \quad \text { indivíduos } \\
\text { maiores de } 30 \text { anos }\end{array}$ & $\begin{array}{l}\text { Os participantes responderam ao } \\
\text { Questionário geral de saúde de } 12 \\
\text { itens (GHQ-12), que avaliou o } \\
\text { sofrimento psicológico d As } \\
\text { associações entre sinais de DTM e } \\
\text { sofrimento psicológico medidos pelo } \\
\text { GHQ-12 foram examinadas em ambos } \\
\text { os sexos. }\end{array}$ & $\begin{array}{l}\text { Nenhuma } \\
\text { proposta }\end{array}$ & $\begin{array}{l}\text { Os sinais de DTM e o sofrimento psicológico parecem } \\
\text { estar associados. }\end{array}$ \\
\hline Lei et al., $2016^{30}$ & 578 adolescentes & $\begin{array}{l}\text { Aplicação de questionários: } \\
\text { Diagnostic for } \quad \text { Criteria } \\
\text { Temporomandibular } \\
\text { Disorders (DC/TMD) Symptom } \\
\text { Questionnaire; } \\
\text { Pittsburgh Sleep Quality Index } \\
\text { (CPSQI), } \\
\text { Depression, Anxiety, and Stress } \\
\text { Scales-21 (DASS-21) }\end{array}$ & $\begin{array}{l}\text { Nenhuma } \\
\text { proposta }\end{array}$ & $\begin{array}{l}\text { A prevalência de sintomas de DTM em adolescentes } \\
\text { asiáticos é alta. O sono perturbado e o sofrimento } \\
\text { psicológico estão correlacionados com DTM. }\end{array}$ \\
\hline $\begin{array}{l}\text { Fernandes- } \\
\text { Azevedo et al., } \\
2017^{31}\end{array}$ & $\begin{array}{ll}105 \text { estudantes de } \\
\text { odontologia }\end{array}$ & $\begin{array}{l}\text { Aplicação de dois questionários: } \\
\text { Research Diagnostic Criteria for } \\
\text { Temporomandibular r Disorders } \\
\text { (RDC/TMD) - para o diagnóstico de } \\
\text { DTM e o nível de ansiedade foi obtido } \\
\text { através do State Trait Anxiety } \\
\text { Inventory. }\end{array}$ & $\begin{array}{l}\text { Nenhuma } \\
\text { proposta }\end{array}$ & $\begin{array}{l}\text { A DTM conjunta foi o subtipo mais prevalente de DTM } \\
\text { em estudantes de odontologia e não foi associada a } \\
\text { níveis de ansiedade. }\end{array}$ \\
\hline Su et al., $2017^{32}$ & $\begin{array}{l}320 \quad \text { pacientes } \\
\text { diagnosticados com } \\
\text { DTM }\end{array}$ & $\begin{array}{l}\text { Uso dos questionários } \\
\text { Checklist-90(SCL-90), } \\
\text { Epwortom } \\
\text { Sleeping Scale (ESS), Questionário de } \\
\text { estresse e Life Orientation Test- } \\
\text { Revised (LOT-R). A DTM foi } \\
\text { quantificada através de scores para a } \\
\text { intensidade de dor. Os fatores } \\
\text { psicológicos e sócio-demográficos } \\
\text { foram correlacionados com a } \\
\text { intensidade da dor e a escala de } \\
\text { deficiência. }\end{array}$ & $\begin{array}{l}\text { Nenhuma } \\
\text { proposta }\end{array}$ & $\begin{array}{l}\text { Entre os fatores psicológicos e sociodemográficos no } \\
\text { presente estudo, } \\
\text { a somatização foi o melhor preditor da intensidade da } \\
\text { dor, enquanto a depressão foi o melhor preditor de } \\
\text { deficiência relacionada à dor, }\end{array}$ \\
\hline
\end{tabular}

\section{DISCUSSÃO}

A contribuição dos fatores psicológicos e psicossomáticos na etiologia, perpetuação e tratamento da DTM é amplamente reconhecida por diversos autores $^{13,15,21,32}$. Entretanto, o levantamento demonstrou a escassez de pesquisas abordando propostas terapêuticas nessa área. As pesquisas citadas no presente trabalho utilizaram em sua maioria TCC, Terapia
Cognitiva-Comportamental como proposta de intervenção ${ }^{11,14,15}$.

A TCC é fundamentada no princípio básico da reestruturação cognitiva, a partir de uma conceituação do paciente e de seus problemas. Inicialmente, tem como objetivo devolver ao paciente a flexibilidade cognitiva, por meio da intervenção sobre as suas cognições, a fim de 
promover mudanças nas emoções e comportamentos que as acompanham.

O tratamento cognitivo comportamental tem eficácia comprovada na DTM ${ }^{14}$. No entanto, a maioria dos pacientes recebe apenas o tratamento dentário sem abarcar as demandas psicológicas muitas vezes presentes nesta disfunção. A TCC resulta em melhorias maiores em habilidades de enfrentamento da dor e tem se mostrado bem aceito pelos pacientes ${ }^{22}$.

Resultados favoráveis também foram encontrados com a TCC associada ao tratamento convencional de DTM e associação à terapia com Amitriptilina ${ }^{11,14,15,22}$. Dentre os benefícios foi descrito auxilio tanto no tratamento da dor quanto no enfrentamento da dor na íntegra, demonstrandose a necessidade da inclusão do tratamento psicológico dentro da clínica de $\mathrm{DTM}^{15,22}$.

Outras formas de técnicas citadas nas abordagens aos pacientes com dor facial crônica ou diagnóstico provável de DTM foi o autorelaxamento. Essa técnica demonstrou ser de fácil aplicação e trouxe resultado positivo sobre a percepção da dor, no entanto, o benefício pode ser maior em pacientes com maiores impactos dos sintomas, tanto disfuncionais quanto adaptativos $^{23}$.

Este levantamento evidencia a importância de uma abordagem multiprofissional na assistência ao paciente com DTM, onde o papel do psicólogo é fundamental no diagnóstico correto e acompanhamento do paciente para o sucesso do tratamento.

Silvola et al. ${ }^{33}$ apontam que o tratamento da DTM, interdisciplinado com a psicoterapia, proporciona um melhor enfrentamento da dor e uma melhoria na qualidade de vida, uma vez que a DTM se estende para todo o contexto social, fisiológico e psicológico. Segundo o autor, a ação do psicólogo se estenderia a todas as dimensões prejudicadas, promovendo assim o restabelecimento da qualidade de vida.

\section{CONCLUSÃO}

O estudo apresentou, por meio de revisão de literatura, necessidade de abordagem integradora para que se possa promover uma melhoria na qualidade bucal e de vida em pacientes com DTM. Sendo assim, mais estudos nesta área proporcionarão chances de novos tratamentos para a DTM, desenvolvendo tratamentos menos invasivos e mais eficientes, não somente por meio da Terapia Cognitivo-Comportamental, mas também em outras terapias psicológicas com potencial a ser explorado.

\section{REFERÊNCIAS}

1. Dworkin SF, LeResche L. Research diagnostic criteria for temporomandibular disorders: review, criteria, examinations and specifications, critique. $\mathbf{J}$ Craniomandib Disord. 1992; 6(4):301-55.

2. Stocka A, Kuc J, Sierpinska T, Golebiewska M, Wieczorek A. The Influence of Emotional State on the Masticatory Muscles Function in the Group of Young Healthy Adults. Biomed Res Int. 2015; 2015: 174013.

3. Schmitter M, Keller G, Giannakopoulos N, Rammelsberg P. Chronic stress in myofascial pain patients. Clin Oral Investig. 2010; 14(5): 593-7.

4. Okeson JP, de Leeuw R. Differential diagnosis of temporomandibular disorders and other orofacial pain disorders. Dent Clin North Am. 2011; 55(1):105-20.

5. Dantas XMA, Santos LJE, Vilela MR, Lucela SBL. Perfil epidemiológico de pacientes atendidos em um
Serviço de Controle da Dor Orofacial. 2015; 44(6):313-9.

6. Cordeiro BI, Guimarães SA. Características dos pacientes com disfunção temporomandibular quanto à queixa principal, sinais, sintomas, sexo e idade. 2012; 60(2): 143-8

7. Silva GMAJ, Dibai Filho VA, Machado AA, Oliveira MEL, Navega TM. Correlação entre autoestima e grau de severidade da disfunção temporomandibular em sujeitos controle e afetados. 2012; 41(6):377-83.

8. John MT, Dworkin SF, Mancl L. A. Reliability of clinical temporomandibular disorder diagnoses. Pain. 2005; 118(1-2):61-9.

9. Motta LJ, Bussadori SK, Godoy CLH, BiazottoGonzalez DA, Martins MD, Silva RS. Disfunção Temporomandibular segundo o Nível de Ansiedade em Adolescentes. Psic Teor e Pesq. 2015; 31(3):389-95.

10. Costa LFM, Guimarães JP. Disfunções temporomandibulares: qual o papel atual do cirurgiãodentista? Rev Bras Odontol. 2002; 59(5):351-4.

11. Litt MD, Shafer DM, Kreutzer DL. Brief cognitivebehavior al treatment for TMD pain: long-term outcomes and moderators of treatment. Pain. 2010; 151(1):110-6.

12. Kuroiwa DN, Marinelli JG, Rampani MS, de Oliveira W, Nicodemo D. Desordens temporomandibulares e dor orofacial: Estudo da qualidade de vida medida pelo Medical Outcomes Study 36 - item short form Health Survey. Revista Dor. 2011; 12(2):93-8.

13. Fillingim RB, Ohrbach R, Greenspan JD, Knott C, Dubner R, Bair E et al. Potential psychosocial risk factors for chronic TMD: descriptive data and empirically identified domains from the OPPERA casecontrol study. J Pain. 2011; 12(11 Suppl):46-60.

14. Calderon PS, Tabaquim MLM, Oliveira LC, Camargo APA, Ramos Netto TC, Conti PCR. Effectiveness of cognitive-behavioral therapy and amitriptyline in patients with chronic temporomandibular disorders: a pilot study. Braz Dent J. 2011; 22(5):415-21.

15. Aggarwal VR, Tickle M, Javidi H, Peters S. Reviewing the evidence: can cognitive behavioral therapy improve outcomes for patients with chronic orofacial pain. J Orofac Pain. 2011; 24(2):163-71.

16. Kim YK, Kim SG, Im JH, Yun PY. Clinical survey of the patients with temporomandibular joint disorders, using Research Diagnostic Criteria (Axis II) for TMD: preliminary study. J Craniomaxillofac Surg. 2012; 40(4):366-72.

17. Miettinen O, Lahti S, Sipiläcorresponding K. Psychosocial aspects of temporomandibular disorders and oral health-related quality-of-life. Acta Odontol Scand. 2012; 70(4):331-6.

18. Diniz MR, Sabadin PA, Leite FP, Kamizaki R. Psychological factors related to temporomandibular disorders: an evaluation of students preparing for college entrance examinations. Acta Odontol Latinoam. 2012; 25(1):74-81.

19. Bezerra BPN, Ribeiro AIAM, Farias ABL, Farias ABL, Fontes LBC, Nascimento SR et al. Prevalência da disfunção temporomandibular e de diferentes níveis de ansiedade em estudantes universitários. Rev Dor. 2012; 13(3):235-42.

20. Zavanelli AC, Zuim PRJ, Barboza GS, Justi MM. Disfunção temporomandibular na visão de profissionais 
e acadêmicos de odontologia. Estudos de Psicologia. 2013; 30(4):553-9.

21. Fillingim RB, Ohrbach R, Greenspan JD, Knott C, Diatchenko L, Dubner R et al. Psychological factors associated with development of TMD: the OPPERA prospective cohort study. J Pain. 2013; 14(12 Suppl):T75-90.

22. Shedden Mora MC, Webber D, Neff A, Rief W. Biofeedback-based cognitive-behavioral treatment compared with occlusal splint for temporomandibular disorder: a randomized controlled trial. Clin J Pain. 2013; 29(12):1057-65.

23. Kirschneck CR, Ömer P, Proff P, Lippold C. Psychologicalprofile and self-administeredrelaxation in patients with craniofacialpain: a prospective in-office study. Head Face Med. 2013; 20:9:31.

24. Wieckiewicz M, Grychowska N, Wojciechowski K, Pelc A, Augustyniak M, Sleboda A et al. Prevalence and correlation between TMD based on RDC/TMD diagnoses, oral parafunctions and psychoemotional stress in Polish university students. Biomed Res Int. 2014; 2014:472346.

25. Roldán-Barraza C, Janko S, Villanueva J, Araya I, Lauer HC. A systematic review and meta-analysis of usual treatment versus psychosocial interventions in the treatment of myofascial temporomandibular disorder pain. J Oral Facial Pain Headache. 2014; 28:205-22.

26. Ismail $F$, Eisenburger $M$, Lange $K$, Schneller $T$, Schwabe L, Strempel $\mathrm{J}$ et al. Identification of psychological comorbidity in TMD-patients. Cranio. 2015; 23:1-9.

27. Costa YM, Porporatti AL, Stuginski-Barbosa J, Bonjardim LR, Conti PC. Additional effect of occlusal splints on the improvement of psychological aspects in temporomandibular disorder subjects: A randomized controlled trial. Arch Oral Biol. 2015; 60(5):738-44.

28. Salameh E, Alshaarani F, Hamed HA, Nassar JA. Investigation of the relationship between psychosocial stress and temporomandibular disorder in adults by measuring salivary cortisol concentration: A casecontrol study. J Indian Prosthodont Soc. 2015; 15(2):148-52.

29. Tuuliainen L, Sipilä K, Mäki P, Könönen M, Suominen AL. Association between clinical signs of temporomandibular disorders and psychological distress among an adult finnish population. J Oral Facial Pain Headache. 2015; 29(4):370-7.

30. Lei J, Fu J, Yap AU, Fu KY. Temporomandibular disorders symptoms in Asian adolescents and their association with sleep quality and psychological distress. Cranio. 2016; 34(4):242-9.

31. Fernandes Azevedo AB, Câmara-Souza MB, Dantas IS, de Resende CMBM, Barbosa GAS. Relationship between anxiety and temporomandibular disorders in dental students. Cranio. 2017; 9:1-4.

32. Su N, Lobbezoo F, van Wijk A, van der Heijden GJ, Visscher CM. Associations of pain intensity and painrelated disability with psychological and sociodemographic factors in patients with temporomandibular disorders: a cross-sectional study at a specialised dental clinic. J Oral Rehabil. 2017; 44(3):187-96.

33. Silvola AS, Tolvanen M, Rusanen J, Sipilä K, Lahti S, Pirttiniemi P. Do changes in oral health-related qualityof-life, facial pain and temporomandibular disorders correlate after treatment of severe malocclusion? Acta Odontol Scand. 2016; 74(1):44-50.

\section{CONFLITO DE INTERESSES}

Os autores declaram não haver conflitos de interesse.

\section{AUTOR PARA CORRESPONDÊNCIA}

\section{Adriana Cristina Zavanelli}

zavanelliac@foa.unesp.br

Submetido em 28/09/2017

Aceito em 09/11/2017 